Atmos. Chem. Phys., 10, 3529-3544, 2010

www.atmos-chem-phys.net/10/3529/2010/

(C) Author(s) 2010. This work is distributed under

the Creative Commons Attribution 3.0 License.

\title{
A review of the theoretical basis for bulk mass flux convective parameterization
}

\author{
R. S. Plant \\ Department of Meteorology, University of Reading, Earley Gate, P.O. Box 243, Reading, RG6 6BB, UK
}

Received: 6 November 2009 - Published in Atmos. Chem. Phys. Discuss.: 23 November 2009

Revised: 2 March 2010 - Accepted: 29 March 2010 - Published: 16 April 2010

\begin{abstract}
Most parameterizations for precipitating convection in use today are bulk schemes, in which an ensemble of cumulus elements with different properties is modelled as a single, representative entraining-detraining plume. We review the underpinning mathematical model for such parameterizations, in particular by comparing it with spectral models in which elements are not combined into the representative plume. The chief merit of a bulk model is that the representative plume can be described by an equation set with the same structure as that which describes each element in a spectral model. The equivalence relies on an ansatz for detrained condensate introduced by Yanai et al. (1973) and on a simplified microphysics. There are also conceptual differences in the closure of bulk and spectral parameterizations. In particular, we show that the convective quasi-equilibrium closure of Arakawa and Schubert (1974) for spectral parameterizations cannot be carried over to a bulk parameterization in a straightforward way. Quasi-equilibrium of the cloud work function assumes a timescale separation between a slow forcing process and a rapid convective response. But, for the natural bulk analogue to the cloud-work function, the relevant forcing is characterised by a different timescale, and so its quasi-equilibrium entails a different physical constraint. Closures of bulk parameterizations that use a parcel value of CAPE do not suffer from this timescale issue. However, the Yanai et al. (1973) ansatz must be invoked as a necessary ingredient of those closures.
\end{abstract}

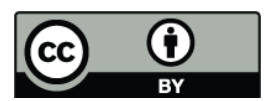

Correspondence to: R. S. Plant (r.s.plant@reading.ac.uk)

\section{Introduction}

The parameterization of precipitating convection for both general-circulation and numerical weather prediction models is a notoriously stubborn problem (e.g. Arakawa, 2004; Randall et al., 2007). The parameterization scheme takes as input the grid-scale flow in the parent model and attempts to deduce from that the tendencies to the resolved-flow arising from complicated, nonlinear sub-grid processes that are imperfectly understood (due to the microphysics for instance), and even imperfectly defined (for example, the convective and boundary-layer parameterizations will often be designed separately and their coupling considered only later). Thus, the task is daunting but nonetheless important in order to obtain satisfactory behaviour from the parent model.

One approach to convective parameterization is to estimate a target atmospheric state produced through the action of convection and to drive the model state towards that target (e.g. Betts and Miller, 1986). Another is more process-oriented, assuming that the "mass flux" in convective "plumes" dominates the upwards transport. Tendencies are calculated from the interactions between simple onedimensional entraining plumes and their environment, together with the effects of compensating subsidence within the environment. The concept of an entraining plume is clearly a great over-simplification of the dynamics and thermodynamics of an individual cloud. However, it does appear tolerably accurate when averaged over many clouds (e.g. Lin and Arakawa, 1997; Kuang and Bretherton, 2006) and so to provide a reasonable basis for parameterization (e.g. Lin, 1999), although possibly only because of a somewhat fortuitous cancellation between errors (Lin and Arakawa, 1997).

Published by Copernicus Publications on behalf of the European Geosciences Union. 
The purpose of this article is to review "bulk" mass flux parameterizations of deep convection and, in particular, to compare their theoretical basis to that of their "spectral" counterparts. It has been argued (e.g. by Esbensen, 1978) that shallow convection should be parameterized separately, not least because different adjustment timescales may apply, and we will not consider such cloud any further here. The model for an individual plume (labelled $i$ ) can be formulated through budget equations which we shall state explicitly in Sect. 2 . Bulk and spectral models are distinguished through the way in which the collective effects of an ensemble of plumes are treated. In a spectral model, plumes are grouped together into types characterised by some parameter, say $\lambda$, so that the mass flux due to plumes of each type can be represented as ${ }^{1}$

$\mathcal{M}(\lambda) d \lambda=\sum_{i \in(\lambda, \lambda+d \lambda)} M_{i}$

A generalization to multiple such parameters is trivial, although not common (Nober and Graf, 2005, is an exception). In a bulk model there is no consideration of plume types and the collective effects are treated through summation over all plumes to produce a single, effective "bulk" or "ensemble" plume. In both types of model, it is assumed that there are sufficient plumes to be treated statistically, such as might be found within a region of space-time "large enough to contain an ensemble of cumulus clouds but small enough to cover only a fraction of a large-scale disturbance" (Arakawa and Schubert, 1974, p. 675). The existence of any such welldefined region in practice is certainly open to question (see e.g. Mapes, 1997), particularly in respect of the roles of spatial and temporal averaging (e.g Yano et al., 2000), but we shall nonetheless proceed with that notion here.

A spectral parameterization certainly requires more computations, with multiple plume types to be explicitly considered. Historically this was an important consideration, and (at least in part) has motivated the development of various bulk parameterizations for operational models (e.g. Tiedtke, 1989; Gregory and Rowntree, 1990; Gregory, 1997; Bougeault, 1985; Gerard and Geleyn, 2005). In recent times, with enhanced computer performance, it is less clear that the run time of a convective parameterization should be quite such a strong consideration in its formulation. Major computational resources are being devoted to model the climate with convection being represented explicitly rather than parameterized (e.g. Khairoutdinov et al., 2005; Garner et al., 2007; Shutts and Allen, 2007). In comparison, the computational overhead of a spectral as opposed to a bulk parameterization is modest indeed.

In parameterizations for mesoscale models another argument has sometimes been advanced for single-plume as opposed to spectral formulations: since the grid elements are relatively small, "it is assumed that all convective clouds in

\footnotetext{
${ }^{1}$ Eq. (78) of Arakawa and Schubert (1974)
}

an element are alike" (Fritsch and Chappell, 1980, p. 1724). This argument (see also Frank and Cohen, 1987; Fritsch and Kain, 1993) fails to recognize that although there may be only a small number of clouds present in a relatively small grid box, the properties of those clouds may not be knowable a priori but rather are randomly drawn from those of the statistical ensemble. As shown by Plant and Craig (2008) then, the consideration of smaller grid boxes actually leads not to a formulation based on a single-plume with prescribed properties but rather to stochastic parameterizations in which a spectral formulation is sub-sampled.

We do not seek here to review the relative performance of bulk and spectral parameterizations, not least because it would be debatable whether any truly clean tests exist. We do, however, revisit and reconsider the mathematical formulation of plume-based models, asking in particular whether a bulk parameterization is a valid simplification of a spectral parameterization in principle. We wish to be very clear about the simplifications, approximations or ansatze required to construct the bulk analogue of a spectral parameterization. It has been recognized by Lawrence and Rasch (2005) for example, that bulk and spectral parameterizations are not completely equivalent representations for the turbulent transport of all quantities, a point that has important implications ${ }^{2}$ for chemical transport. Our attention here though is restricted to moisture and thermodynamic transports.

As our exemplar spectral formulation, we use the wellknown scheme of Arakawa and Schubert (1974, hereinafter AS74). As our exemplar bulk formulation, we use the scheme of Yanai et al. (1973, hereinafter YEC73). This is not a parameterization as such, rather a system for diagnostic analyses. However, the rationale for bulk parameterizations in the literature does seem to be by appeal to the YEC73 system. This is stated explicitly by Gregory and Rowntree (1990) and Gregory (1997) for example.

The remainder of this article is structured as follows. Budget equations for individual plumes are given in Sect. 2 and assumptions about detrainment are discussed in Sect. 3. It is at that stage that the bulk and spectral formulations first depart and the implications for determining the collective effects of the cloud ensemble are explained in Sect. 4. Section 5 introduces the concept of a normalization transformation, which will be useful when we proceed to discuss closure in Sect. 6. Conclusions are presented in Sect. 7.

\footnotetext{
${ }^{2}$ For example, an additional, and somewhat arbitrary, parameter appears in tracer transport calculations when a spectral parameterization is approximated by a bulk plume (Lawrence and Rasch, 2005, Sect. $2 \mathrm{c}(1))$.
} 


\section{Plume equations}

The budget equations for a single entraining plume are given $^{3}$ in AS74, specifically

$$
\begin{aligned}
& \frac{\partial \rho \sigma_{i}}{\partial t}=E_{i}-D_{i}-\frac{\partial M_{i}}{\partial z} \\
& \frac{\partial \rho \sigma_{i} s_{i}}{\partial t}=E_{i} \bar{s}-D_{i} s_{D i}-\frac{\partial M_{i} s_{i}}{\partial z}+L \rho c_{i}+\rho Q_{\mathrm{R} i} \\
& \frac{\partial \rho \sigma_{i} q_{i}}{\partial t}=E_{i} \bar{q}-D_{i} q_{D i}-\frac{\partial M_{i} q_{i}}{\partial z}-\rho c_{i} \\
& \frac{\partial \rho \sigma_{i} l_{i}}{\partial} t=-D_{i} l_{D i}-\frac{\partial M_{i} l_{i}}{\partial z}+\rho c_{i}-R_{i}
\end{aligned}
$$

A complete guide to the nomenclature used in this article can be found in the Appendix. $i$ labels a plume, which occupies a fractional area of $\sigma_{i} . s=c_{p} T+g z$ is the dry static energy, $Q_{\mathrm{R}}$ the radiative heating rate, $R$ the rate of conversion of liquid water to precipitation and $c$ the rate of condensation. $E_{i}$ and $D_{i}$ are respectively the entrainment and detrainment rates. In writing the entrainment terms on the right-hand side of the above equations it has been assumed ${ }^{4}$ that $\widetilde{q} \approx \bar{q}$ and $\tilde{s} \approx \bar{s}$, where the overbar denotes horizontal averaging and the tilde denotes a quantity evaluated within the cloud-free environment, which is assumed homogeneous. In writing the detrainment terms the subscript $D i$ denotes a value on detrainment from the $i$-th plume. Detrainment occurs only in a thin layer at the plume top, and it should be understood that $E_{i}=0$ in the detrainment layer and that $D_{i}=0$ elsewhere. Although some authors (e.g. Johnson, 1977; McBride, 1981) have experimented with simple representations of detrainment throughout the depth of individual plumes, the effects seem to be modest.

The above equations also include the mass flux ${ }^{5}$

$M_{i}=\rho \sigma_{i} w_{i}$

The effects of the plumes on their environment can be represented very simply under the usual mass flux approximations of $\widetilde{w} \ll w_{i}$ and $\sigma_{i} \ll 1$. For some intensive variable $\chi$ we have ${ }^{6}$

$\rho \overline{\chi^{\prime} w^{\prime}}=\sum_{i} M_{i}\left(\chi_{i}-\tilde{\chi}\right)$

The prime denotes a local deviation from the horizontal mean. It should be recalled that a mass-flux representation

\footnotetext{
${ }^{3}$ Their Eqs. (43) to (50). Note that we have made some changes of notation from AS74 in order to assist in the comparison with YEC73. Specifically $C_{i} \rightarrow \rho c_{i}, \mathcal{E} \rightarrow \bar{e}$ and $Q_{\mathrm{R}}(\mathrm{AS} 74) \rightarrow \rho Q_{\mathrm{R}}$ (YEC73).

${ }^{4}$ Nordeng (1994, p. 11) argues that the usual assumptions for the source of entrained air will tend to overestimate dilution in the deepest plumes.

${ }^{5}$ Eq. (2) of AS74

${ }^{6}$ cf. Eq. (23) of YEC73, or Eqs. (35) and (36) of AS74
}

for the vertical fluxes, although extensively used in convective parameterization and in diagnostic studies, is not without its difficulties (e.g. Swann, 2001; Yano et al., 2004).

The above equations do not describe mesoscale circulations (e.g. Yanai and Johnson, 1993), downdrafts (e.g. Johnson, 1976) or phase changes involving ice (e.g Johnson and Young, 1983). These are, of course, considerable limitations for practical applications, but not important for our present purposes. Should a bulk parameterization without downdrafts (say) prove to be an ill-defined simplification of a spectral parameterization then it would not become well-defined through the addition of downdraft terms.

The equivalent equation set ${ }^{7}$ in YEC73 differs from that above in that:

i. time derivative terms are omitted; ${ }^{8}$

ii. AS74's approximation $\tilde{\chi} \rightarrow \bar{\chi}$ is not made in the entrainment terms;

iii. values on detrainment are assumed identical to the inplume values, $\chi_{D i}=\chi_{i}$.

Although it is not necessary to do so, both YEC73 and AS74 simplify the radiative heating in Eq. (3): YEC73 by neglecting in-cloud radiation and AS74 by neglecting this within the entrainment layer. ${ }^{9}$

Assuming the in-plume air to be saturated at and above cloud base leads to ${ }^{10}$

$$
\begin{aligned}
& s_{i}-\bar{s} \approx \frac{1}{1+\gamma}\left(h_{i}-\bar{h}^{*}\right) \\
& L\left(q_{i}-\bar{q}^{*}\right) \approx \frac{\gamma}{1+\gamma}\left(h_{i}-\bar{h}^{*}\right)
\end{aligned}
$$

where

$\gamma=\left.\frac{L}{c_{p}} \frac{\partial \bar{q}^{*}}{\partial \bar{T}}\right|_{\bar{p}}$

Here $h=s+L q$ is the moist static energy and the star denotes saturation ${ }^{11}$. The same equations appear ${ }^{12}$ in YEC73, albeit with $\bar{\chi}$ replaced by $\tilde{\chi}$.

\footnotetext{
${ }^{7}$ Their Eqs. (27) to (30)

${ }^{8}$ Such terms are later dropped by AS74. Cho (1977) considered the effects of incorporating a plume lifecycle into a mass-flux ensemble framework, and showed that the effects on the apparent heating $Q_{1}$ are negligible. However, an additional contribution arises to the apparent moisture sink $Q_{2}$ (their Eqs. 38 and 39) due to the mixing of air from the decaying plume with its environment. Budget diagnosis suggests that this may be significant near cloud base, but is less important elsewhere. See also Grell et al. (1991, p. 26).

${ }^{9}$ See the sentence after their Eq. (86).

${ }^{10}$ Eqs. (55) to (57) of AS74

${ }^{11}$ Notice that $\bar{\chi}^{*}$ is to be interpreted as the saturation value that corresponds to $\bar{T}$ and $\bar{p}$ : it is not the same as $\overline{\chi^{*}}$, the horizontal average of the local saturation values.

${ }^{12}$ As their Eqs. (33) and (34)
} 


\section{Detrainment assumptions}

It is in the detrainment assumptions that the spectral and bulk formulations depart in a significant way. Let us consider each formulation in turn.

\subsection{Detrainment in AS74}

A thin detrainment layer at the top of each plume occurs at its level of neutral buoyancy, denoted $\widehat{z}_{i}$. All plumes detraining at a given level are assumed to have the same in-plume liquid water there ${ }^{13}, \widehat{l} \equiv l_{i}\left(\widehat{z}_{i}\right)$. Note that a distinction is drawn between $\widehat{l}$ and the detrained liquid water $l_{D i}$ on the grounds that "additional condensation (or evaporation) may be taking place near cloud top due to concentrated radiational cooling (or heating) there" (AS74, p. 680). ${ }^{14}$

The neutral-buoyancy condition is the equality of the environmental and the in-plume virtual dry static energy, which can also be expressed as, ${ }^{15}$

$h_{i}\left(\widehat{z}_{i}\right) \equiv \widehat{h}^{*}=\bar{h}^{*}+h_{\mathrm{vc}}$

where the virtual contribution is given by

$h_{\mathrm{vc}}=-\frac{(1+\gamma) L \epsilon}{1+\gamma \epsilon \delta}\left(\delta\left(\bar{q}^{*}-\bar{q}\right)-\widehat{l}\right)$

with

$\epsilon=\frac{c_{p} \bar{T}}{L}$

and $\delta=0.608$. Simplification of the budget equations in the detrainment layer also produces detrainment relations for other variables, specifically ${ }^{16}$

$$
\begin{aligned}
& D_{i} s_{D i}=D_{i} \widehat{s}+L \rho c_{i}+\rho Q_{\mathrm{R} i} \\
& D_{i} q_{D i}=D_{i} \widehat{q}^{*}-\rho c_{i} \\
& D_{i} l_{D i}=D_{i} \widehat{l}+\rho c_{i}
\end{aligned}
$$

where $^{17}$

$\widehat{s} \equiv \bar{s}+s_{\mathrm{vc}} ; \widehat{q}^{*} \equiv \bar{q}^{*}+q_{\mathrm{vc}}$

with virtual contributions of

$s_{\mathrm{vc}}=-\frac{L \epsilon}{1+\gamma \epsilon \delta}\left(\delta\left(\bar{q}^{*}-\bar{q}\right)-\widehat{l}\right) ;$

$q_{\mathrm{vc}}=-\frac{\gamma \epsilon}{1+\gamma \epsilon \delta}\left(\delta\left(\bar{q}^{*}-\bar{q}\right)-\widehat{l}\right)$

Although it is not stated explicitly, AS74 neglect precipitation formation in the detrainment layer, and so omit a term $-R_{i}$ from the right-hand-side of Eq. (16).

\footnotetext{
${ }^{13}$ Later in their derivation, AS74 choose to use a single spectral parameter defining the entrainment, $\lambda$, such that $\widehat{z}$ and $\lambda$ are monotonic functions of each other. This assumption for $\widehat{l}$ is then required for consistency with that choice.

${ }^{14}$ See our Eq. (16) below for the mathematical statement of this.

${ }^{15}$ Eqs. (63) and (64) of AS74

${ }^{16}$ These are consistent with Eqs. (68) and (69) of AS74.

17 cf. Eqs. (72) and (73) of AS74
}

\subsection{Detrainment in YEC73}

In order to formulate the detrainment assumptions of YEC73, we must first introduce the mass-flux-weighting operation, ${ }^{18}$ defined by

$\chi_{B}=\frac{\sum_{i} M_{i} \chi_{i}}{\sum_{i} M_{i}}$

which produces a "bulk" value of $\chi$.

At the heart of bulk models is an ansatz that the liquid water detrained from each individual plume is given by the bulk value, ${ }^{19}$

$l_{D i}=l_{i}=l_{B}$

The relation is described by YEC73 (p. 615) as being a "gross assumption" but "needed to close the set of equations". Its practical importance is not clear from the literature ${ }^{20}$. Figure 1 illustrates the use of the ansatz. 200 entraining plumes are launched into Jordan's (1958) "mean hurricane season" sounding, each with the same arbitrary mass flux at the updraft base and with a range of entrainment rates that result in detrainment levels between 850 and $150 \mathrm{mb}$. Examples of the mass flux and liquid water profiles for some of those plumes are shown in Fig. 1, along with the profiles of $l_{D i}$ and $l_{B}$. Two effects of the ansatz are appparent. First, that it will detrain liquid water at levels in between the lifting condensation level and the detrainment level of the most strongly entraining plume in the ensemble: here between 950 and $850 \mathrm{mb}$. Second, that liquid-water detrainment is systematically overestimated by the ansatz: here by $\sim 20 \%$. At any given level, the detraining plumes have lower liquid-water contents than the plumes which remain buoyant. Thus, any procedure for averaging over plumes must produce a bulk value larger than the actual detraining liquid-water.

Another point of difference between YEC73 and AS74 is that the detrainment level is defined by YEC73 to be the height $\widehat{z}_{i}$ at which ${ }^{21}$

$h_{i}\left(\widehat{z}_{i}\right)=\widetilde{h}^{*}$

\footnotetext{
${ }^{18}$ YEC73 used a double overbar to denote this operation.

${ }^{19}$ Eq. (39) of YEC73

${ }^{20}$ Yanai et al. (1976) compared results from bulk and spectral diagnostic models using data from the Marshall Islands. At least in terms of the profiles of total mass flux and detrainment flux, differences were modest. However, the comparison is complicated by "data corrections" made for the spectral but not for the bulk analysis (Yanai et al., 1976, Sect. 3), and the remarks of Tiedtke (1989, p. 1781) on this matter still hold today: it is difficult to know how well such comparisons might hold more generally.

${ }^{21}$ Eq. (38) of YEC73. Although YEC73 claim that the same assumption is used in a version of AS74 (referenced by YEC73 as 1973 and with a status of "to be published"), we must presume there were changes in producing the final version of AS74, as the neutralbuoyancy conditions are clearly not identical.
} 
from which it follows that

$s_{i}\left(\widehat{z}_{i}\right)=\widetilde{s} ; T_{i}\left(\widehat{z_{i}}\right)=\widetilde{T} ; q_{i}\left(\widehat{z}_{i}\right)=\widetilde{q}^{*}$

In this definition, "the virtual temperature correction has been neglected for simplicity" (Yanai et al., 1976, p. 978). It should be recognized, however, that the virtual contribution cannot be accounted for fully within a bulk model since it involves the in-plume liquid water $\widehat{l}$ (Eqs. 12 and 19). The virtual contribution is not often discussed. Johnson (1976, p. 1894) noted that $h_{\mathrm{vc}} / \widetilde{h}^{*} \ll 1$ and so the virtual term could reasonably be neglected in YEC73. However, Nordeng's (1994, p. 25) experiments with a bulk parameterization found some significant effects from changing the detrainment condition used.

It is straightforward to evaluate the specific-humidity component of $h_{\mathrm{vc}} / \widetilde{h}^{*}$ explicitly, and for the Jordan (1958) sounding we find that the ratio has a maximum amplitude of around 0.005 in the lower troposphere. Moreover, $s_{\mathrm{vc}} \widehat{s}$ and $q_{\mathrm{vc}} / \widehat{q}^{*}$ peak at 0.002 and 0.04 , respectively, so that it would appear reasonable to neglect virtual effects as being small corrections to in-plume variables in the detrainment layer. However, the purpose of Eq. (11) or (21) is to determine $\widehat{z}_{i}$ and Fig. 2a shows that the environmental gradient of saturated moist static energy is small in the tropical upper troposphere. Hence, even small errors in the specification of the neutral-buoyancy condition could result in considerable errors in a calculation of cloud top. Such errors are difficult to estimate reliably when $\partial \widetilde{h}^{*} / \partial z$ is small, particularly if there is any noise in the $\widetilde{h}^{*}$ sounding data. For this reason, in Fig. $2 \mathrm{~b}$ we plot the quantity

$\Delta \widehat{z}=\frac{1}{2}\left\{\left|\frac{s_{\mathrm{vc}}}{\partial \widetilde{s} / \partial z}\right|+\left|\frac{q_{\mathrm{vc}}}{\partial \widetilde{q}^{*} / \partial z}\right|\right\}$

which provides a simple-minded estimate of the effect of virtual contributions on the evaluation of cloud top, and which should be reliable for plumes terminating in the lower troposphere. The corrections are $\sim 150 \mathrm{~m}$.

Of course, one could choose to formulate a bulk model with an estimated virtual correction included, leading to Eqs. (11) and (17) but with $\widehat{l} \rightarrow l_{B}$ (cf. Nordeng, 1994). This would be an improvement if $\left|l_{B}-\widehat{l}\right|<\left|\delta\left(\bar{q}^{*}-\bar{q}\right)-\widehat{l}\right|$

\section{Construction of bulk budget}

We are now in a position to consider the collective effects of the plume ensemble. In Sect. 4.1 we describe YEC73's construction of a bulk plume, and proceed in Sect. 4.2 to compare that to a construction from AS74's equation set.

\subsection{Construction in YEC73}

Budget equations for a representative bulk plume are obtained in YEC73 by summing over plumes in Eqs. (2) to (5).
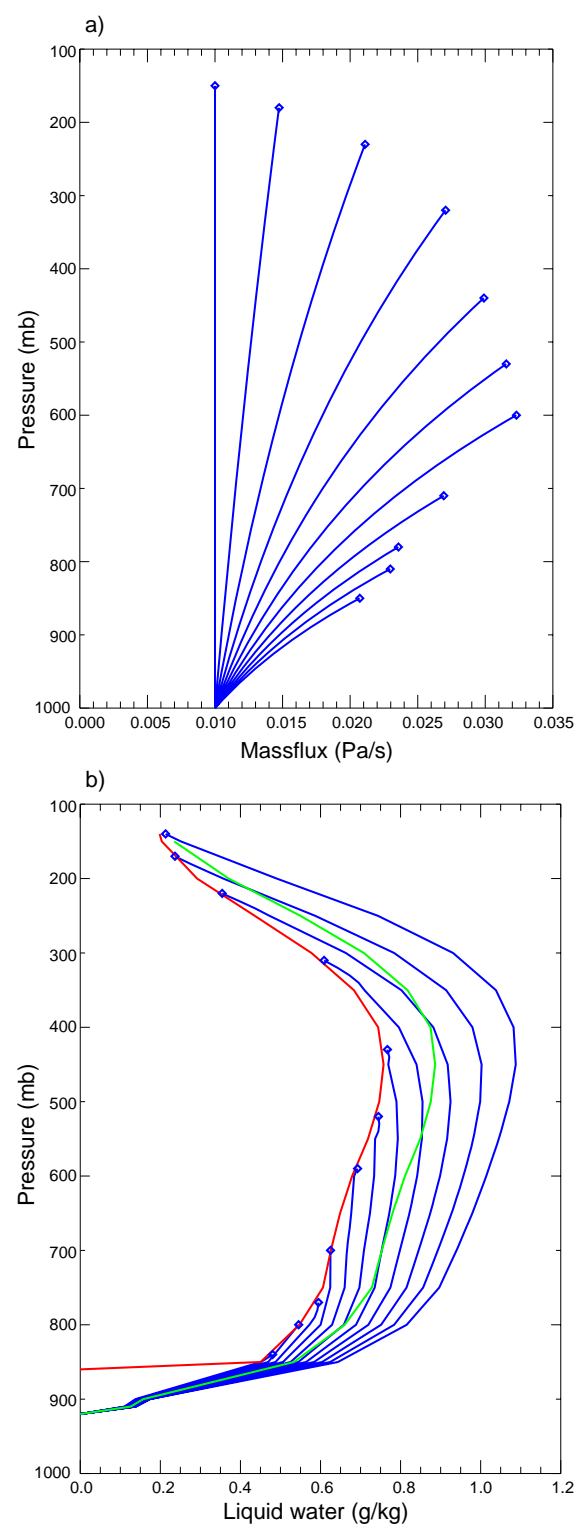

Fig. 1. (a) Vertical profiles of mass flux for entraining plumes launched into the Jordan (1958) sounding. Each plume has an arbitrary updraft-base mass flux of $0.01 \mathrm{Pas}^{-1}$ and a range of entrainment rates are used to produce a range of detrainment levels, these being indicated by the diamond symbol. (b) The corresponding profiles of plume liquid water (blue lines). Also shown are the profiles of detrained liquid water (red line) and the bulk liquid water for the plume ensemble (green line).

Recalling also points (i) to (iii) from Sect. 2 and the detrainment assumptions of Sect. 3.2 then we obtain

$E-D-\frac{\partial M}{\partial z}=0$

$E \widetilde{s}-D \widetilde{s}-\frac{\partial M s_{B}}{\partial z}+L \rho \bar{c}=0$ 

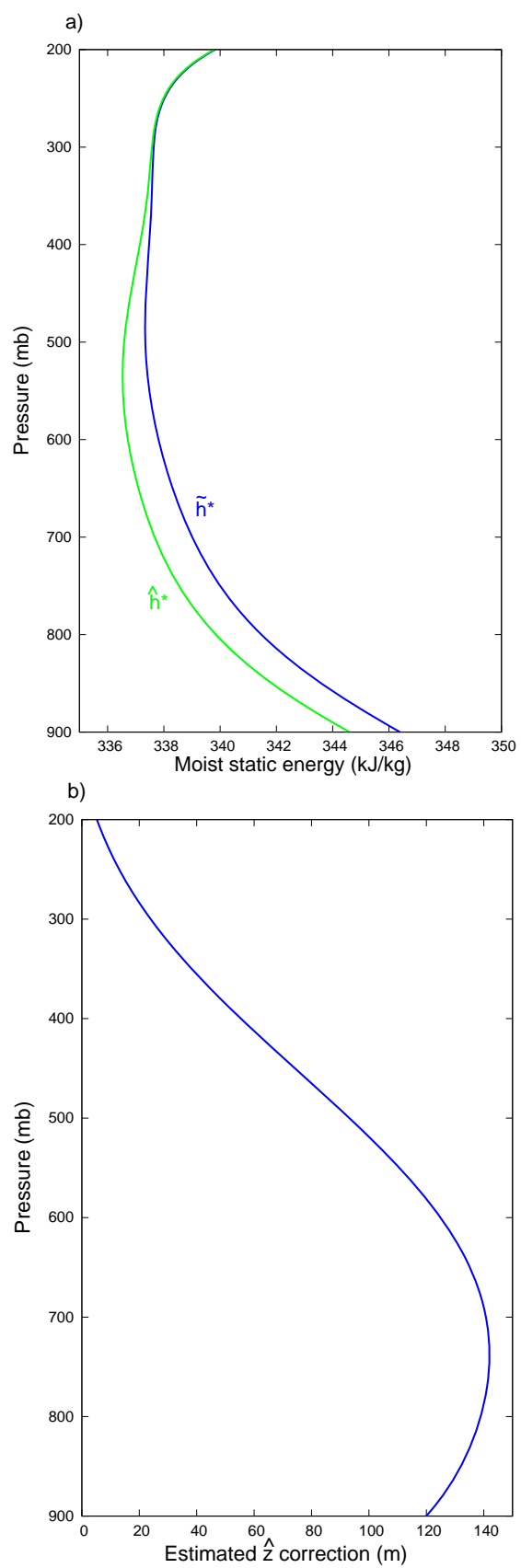

Fig. 2. (a) Vertical profiles of $\widetilde{h}^{*}$ (blue line) and $\widehat{h}^{*}$ (green line; Eq. 11) for the Jordan (1958) sounding. (b) Error in the calculation of $\widehat{z}$, as discussed in the main text and estimated from Eq. (23).

$E \widetilde{q}-D \widetilde{q}-\frac{\partial M q_{B}}{\partial z}-\rho \bar{c}=0$

$-D l_{B}-\frac{\partial M l_{B}}{\partial z}+\rho \bar{c}-R=0$

where

$E=\sum_{i} E_{i} ; D=\sum_{i} D_{i} ; M=\sum_{i} M_{i} ; R=\sum_{i} R_{i}$
Other relevant equations for the bulk plume can be obtained by taking Eqs. (8) and (9), multiplying by $M_{i}$, summing over clouds and then dividing by $M$. This gives

$s_{B}-\bar{s} \approx \frac{1}{1+\gamma}\left(h_{B}-\bar{h}^{*}\right)$

$L\left(q_{B}-\bar{q}^{*}\right) \approx \frac{\gamma}{1+\gamma}\left(h_{B}-\bar{h}^{*}\right)$

The mass-flux approximation for the turbulent flux of $\chi$ (Eq. 7) now reads

$\rho \overline{\chi^{\prime} w^{\prime}}=M\left(\chi_{B}-\tilde{\chi}\right)$

There are also two microphysical relations. ${ }^{22}$ The evaporation term is

$\bar{e}=D l_{B}$

which is simply obtained from a sum over plumes of ${ }^{23}$

$e_{i}=D_{i} l_{i}$

The precipitation rate, summed over the full plume ensemble, is parameterized as the product of $l_{B}$ with an empirical function of height ${ }^{24}$

$R=k(p) l_{B}$

This completes the equation set for the YEC73 bulk model.

\subsection{Construction from AS74}

The model of YEC73 does not provide a complete description of individual entraining plumes. Rather, it posits detrainment conditions using bulk quantities, and so Sect. 4.1 does not make plain the relationship between bulk and spectral models. Here we will construct a bulk plume starting from the description of individual plumes in AS74.

Starting from Eqs. (2) to (5), we set the time derivatives to zero and sum over plumes to obtain

$$
\begin{aligned}
& E-D-\frac{\partial M}{\partial z}=0 \\
& E \bar{s}-\sum_{i} D_{i} s_{D i}-\frac{\partial M s_{B}}{\partial z}+L \rho \bar{c}+\rho \sum_{i} Q_{R i}=0 \\
& E \bar{q}-\sum_{i} D_{i} q_{D i}-\frac{\partial M q_{B}}{\partial z}-\rho \bar{c}=0 \\
& -\sum_{i} D_{i} l_{D i}-\frac{\partial M l_{B}}{\partial z}+\rho \bar{c}-R=0
\end{aligned}
$$

\footnotetext{
${ }^{22}$ Equivalent to Eqs. (47) and (48) of YEC73.

${ }^{23}$ Eq. (31) of YEC73

${ }^{24} R$ must scale with the strength of the convection occurring, and so the "empirical function" must be scaled similarly: cf. Eq. (45) for the AS74 formulation. More formally, in the language of Sect. 5, $k(p)$ is a globally-rescaled quantity.
} 
The next step is to apply the detrainment conditions for individual plumes from Sect. 3.1. Substituting from Eqs. (14) to (16) leads to

$$
\begin{aligned}
& E \bar{s}-D \widehat{s}-\frac{\partial M s_{B}}{\partial z}+L \rho c^{\mathrm{e}}=0 \\
& E \bar{q}-D \widehat{q}^{*}-\frac{\partial M q_{B}}{\partial z}-\rho c^{\mathrm{e}}=0 \\
& -D \widehat{l}-\frac{\partial M l_{B}}{\partial z}+\rho c^{\mathrm{e}}-R=0
\end{aligned}
$$

where we have introduced the superscript e to denote a quantity which is summed only within the entraining layers of contributing plumes. Shortly we shall also use an analogous superscript $d$ to denote a quantity summed only within the detraining layers.

Equations (29) and (30) from the YEC73 bulk system also apply here, as does the mass flux relationship of Eq. (31).

The microphysical equation for evaporation in AS74 is, ${ }^{25}$

$\bar{e}=\sum_{i} e_{i}=\sum_{i} D_{i} l_{D i}$

which can be rewritten as

$\bar{e}=D \widehat{l}+\rho c^{d}$

while the rain rate is parameterized as ${ }^{26}$

$R_{i}=C_{0} M_{i} l_{i}$

where $C_{0}$ is a constant. Hence,

$R=C_{0} M l_{B}$

Clearly the microphysics is extremely simple. Hack et al. (1984) argued that a straightforward improvement would be to set $C_{0}$ differently for deep and shallow clouds. ${ }^{27}$ But notice that if $C_{0} \rightarrow C_{i}$ in Eq. (44) then the simple formula in Eq. (45) can no longer be constructed for a bulk formulation. Rather, some knowledge of the partitioning of $l_{B}$ across the spectrum would be required. Indeed, this is a good example of a general point about the use of more-complicated representations for individual plumes. In general these will only be well-defined within a spectral formulation, and in essence a bulk formulation is committed to crude microphysics.

Of course, the inclusion of fully realistic microphysics in any mass-flux-based convective parameterization is a difficult issue, since microphysical processes have complex, nonlinear dependencies on vertical velocity (e.g. Straka, 2009). Thus, it is no longer sufficient to consider the mass flux alone, but rather the fractional area and vertical velocity must be known separately, which entails carrying an additional equation (e.g Piriou et al., 2007). A spectral formulation is the natural structure for any such attempt, since the averaging inherent in a bulk formulation would not allow one to capture the nonlinearities.

\footnotetext{
${ }^{25}$ Their Eq. (40)

${ }^{26}$ See Eqs. (78), (86) and Appendix B (p. 697, statement between Eqs. B6 and B7) of AS74.

${ }^{27}$ See their Fig. 3.
}

\subsection{Comparison of bulk budgets}

It may be helpful at this stage to highlight the differences between the two bulk-model equation sets from Sects. 4.1 and 4.2.

1. In the dry-static-energy (Eqs. 25 and 39) and moisture budgets (Eqs. 26 and 40) the differences are:

(a) entrainment of $\bar{s}(\bar{q})$ for the AS74 model and of $\widetilde{s}$ $(\widetilde{q})$ for the YEC73 model.

(b) detrainment of $\widehat{s}\left(\widehat{q}^{*}\right)$ for the AS74 model and of $\widetilde{s}(\widetilde{q})$ for the YEC73 model. This arises because YEC73 neglect virtual effects in the detrainment condition. Note that $\widehat{s}\left(\widehat{q}^{*}\right)$ is a function of both large-scale (overbarred) variables and of the nonbulk, in-plume variable $\widehat{l}$ (Eqs. 17 and 19).

(c) condensation within the detrainment layer is explicit in the YEC73 model, but implicit in the AS74 model (because $\widehat{s} \neq s_{D i}$ and $\widehat{q}^{*} \neq q_{D i}$ ).

2. In the liquid-water budgets (Eqs. 27 and 41) the differences are:

(a) detrainment of $\widehat{l}$ for the AS74 model and of $l_{B}$ for the YEC73 model. ${ }^{28}$ Knowledge of $\widehat{l}(z)$ requires knowledge of the plume spectrum because for each height $z$ it has to be determined by integrating the budget equations for an individual plume that detrains at $\widehat{z}_{i}=z$.

(b) condensation within the detrainment layer is explicit in the YEC73 model, but implicit in the AS74 model (because $\widehat{l} \neq l_{D i}$ ).

3. In the YEC73 model, precipitation is related to an empirical function of height, whereas in the AS74 model this function is specified as the product of a constant and the total mass flux (Eqs. 34 and 45).

4. Both models evaporate in-plume water at its detrainment level (Eqs. 32 and 43), but the rate is affected by the assumptions on condensation at this level.

\section{Normalization transformations}

The YEC73 bulk model is designed for diagnostic use and no closure is required. The spectral model of AS74 can also be used in the same way (e.g. Nitta, 1975). However, if a

\footnotetext{
${ }^{28}$ We do not consider downdrafts here, which are significant in diagnostic studies (e.g. McBride, 1981), but it is worth noting that their formulation requires fewer ad hoc assumptions if one uses AS74's determination of detraining liquid water. Compare Johnson (1976) and Nitta (1977), and see in particular p. 1166 of the latter.
} 
bulk or a spectral model is to form the basis of a parameterization then it will require closure. Starting from some first guess for $\mathcal{M}\left(z_{\text {base }}, \lambda\right)$, the closure is essentially the process of rescaling that guess to obtain the actual amount of convective transport. More formally, the rescaling can be thought of as selecting a privileged member from the set of possible normalization transformations. Before proceeding to assess particular closure methods in Sect. 6, it is convenient to define such transformations explicitly and to set out the possible responses of relevant variables.

Normalization transformations, $\mathcal{T}$, are applied to the spectral groupings of Eq. (1). The transformation is a positivelyvalued rescaling of the updraft-base mass flux for each plume sub-ensemble (or loosely, each cloud type),

$\mathcal{M}\left(z_{\text {base }}, \lambda\right) \rightarrow \mathcal{M}\left(z_{\text {base }}, \lambda\right) \mathcal{T}(\lambda)$

where $z_{\text {base }}$ denotes the updraft base, at the top of the mixed layer. Note that this is distinct from the cloud-base, which we denote as $z_{\mathrm{c}}(\lambda)$, and from the lifting condensation level, $z_{\mathrm{LCL}} \equiv z_{\mathrm{c}}(0)$. A subset of normalization transformations of particular interest comprises those for which $\mathcal{T}$ is independent of $\lambda$, which we will refer to as global transformations.

The importance of normalization transformations arises in considerations of possible timescale separations. A time-evolution operator describing changes in the plumeensemble between any two times can always be represented as a normalization transformation. We therefore assert that distinct, well-defined responses to a normalization transformation constitute distinct, well-defined timescales characterizing the ensemble.

All of the variables, $\mathcal{V}$, used in this article transform in one of the following ways.

1. Normalization-invariant variables are unaffected by a normalization transformation, $\mathcal{V} \rightarrow \mathcal{V} \forall \mathcal{T}$. Such variables may be directly dependent on plume dynamics (e.g., $s_{i}$ ), but only through intensive properties of each plume type. They must be independent of the overall amount of convective transport (i.e., of $M$ and $D$ ), and also of its distribution across the plume spectrum (i.e., of $\left.\mathcal{M}\left(z_{\text {base }}, \lambda\right) / M\left(z_{\text {base }}\right)\right)$. They evolve only in response to changes in the large-scale state (i.e., the overbarred variables), which occur on a large-scale timescale of $\tau_{\mathrm{LS}}$

2. Globally-invariant variables are unaffected by a global transformation, so that $\mathcal{V} \rightarrow \mathcal{V}$ if and only if $\mathcal{T}$ is independent of $\lambda$. Such variables are independent of the overall amount of convective transport but are sensitive to its distribution across the plume spectrum. Their evolution is governed by the timescale $\tau_{\text {spec }}$, characterising changes to the spectral distribution under a fixed largescale condition.

3. Normalization-rescaled variables transform as $\mathcal{V} \rightarrow \mathcal{V} \mathcal{T}(\lambda) \forall \mathcal{T}$. Such variables transform alongside one part of the spectrum only, depending extensively on a given plume type $\lambda$, but being independent of the rest of the spectrum. They evolve in response to changes in the particular plume type, which can be characterised by a timescale $\tau_{\lambda}$. The timescale must be at least as long as the corresponding plume lifetime because normalization-rescaled terms such as $\partial \rho \sigma_{i} / \partial t$ were filtered out from Eqs. (2)-(5) in Sect. 2.

4. Globally-rescaled variables transform as $\mathcal{V} \rightarrow \mathcal{V} \mathcal{T}$ if and only if $\mathcal{T}$ is independent of $\lambda$. Such variables depend extensively on the overall amount of convective transport and are sensitive to its distribution across the plume spectrum. Their evolution is governed by the timescale $\tau_{\text {adj }}$ introduced by AS74: if all forcing for convection were to be removed then the overall convective transport would decay on this timescale.

It may be helpful to clarify the meaning of some of the timescales by considering the limiting case of a stepchange in the large-scale forcing, the forcing being held fixed on either side of the step (as in Cohen and Craig, 2004). Mass fluxes associated with specific plume types respond to the step with their specific timescales $\tau_{\lambda}$, but the overall convective transport, as measured by $M\left(z_{\text {base }}\right)$, will approach a new, steady value on the timescale $\tau_{\text {adj }}$. However, a more complete adjustment, with the spectral distribution $\mathcal{M}\left(z_{\text {base }}, \lambda\right) / M\left(z_{\text {base }}\right)$ also required to approach a new steady state, will require a timescale $\tau_{\text {spec }}$. To the best of this author's knowledge, there is no information available from the literature that would provide good estimates of $\tau_{\text {spec }}$ and its possible dependencies. However, it would not appear overly difficult to devise idealized CRM simulations with a view to identifying such a timescale. ${ }^{29}$ We shall show that the timescale is relevant for the closure of bulk mass flux parameterizations.

\section{Closure}

To close a parameterization, some additional physical constraints are imposed which determine the amplitude and spectral distribution of the plume ensemble. As described in Sect. 5 the calculation is performed by rescaling a first guess, and the physical constraints must therefore serve as a generator for the privileged normalization transformation defining the rescaling. For a bulk parameterization, a global transformation is sufficient to provide the rescaling, the spectral distribution being implicit in the choice of $E(z)$.

\footnotetext{
${ }^{29}$ For a smoothly-varying forcing, adjusted, steady values may not be clear. Measures of the lag-correlation between the forcing and $\mathcal{M}\left(z_{\text {base }}, \lambda\right), M\left(z_{\text {base }}\right)$ and $\mathcal{M}\left(z_{\text {base }}, \lambda\right) / M\left(z_{\text {base }}\right)$ could then be used to determine timescales $\tau_{\lambda}$, $\tau_{\text {adj }}$ and $\tau_{\text {spec }}$, respectively (cf. Xu and Randall's (1998) determination of $\tau_{\mathrm{adj}}$ ).
} 
In order for a bulk and a spectral model to be capable of providing equivalent parameterizations, there are two necessary conditions that we can demand of the closures used:

1. given the generator of a normalization transformation that can be computed for the spectral model, it must be possible to construct from that a generator capable of providing a global transformation that can be computed for the bulk model. Such a global transformation could be used to close the bulk model.

2. the generator of the global transformation that closes the bulk model must respect all the same physical constraints that were specified in order to formulate the generator for closure of the spectral model.

Below we describe the AS74 spectral-model closure (Sect. 6.1) and then investigate whether it is possible to develop an equivalent closure for the corresponding bulk model which meets the conditions above (Sects. 6.2 and 6.3). In Sect. 6.4 we discuss other closure methods in the literature.

\subsection{The AS74 closure}

The AS74 closure starts from the following equation ${ }^{30}$ for the kinetic energy $\mathcal{K}$ of a sub-ensemble of plumes

$\frac{\partial \mathcal{K}(\lambda)}{\partial t}=A(\lambda) \mathcal{M}\left(z_{\text {base }}, \lambda\right)-\mathcal{D}(\lambda)$

where $\mathcal{D}$ is the dissipation. $A$ is known as the cloud work function, and is given by the integrated in-plume buoyancy, ${ }^{31}$

$A(\lambda) \equiv \int_{z_{\text {base }}}^{z_{D}(\lambda)} \frac{g}{c_{p} \bar{T}} \frac{\mathcal{M}(z, \lambda)}{\mathcal{M}\left(z_{\text {base }}, \lambda\right)}\left(s_{\mathrm{vp}}(\lambda)-\overline{s_{v}}\right) d z$

Here $z_{D}$ is the detrainment level, $s_{v}=c_{p} T_{v}+g z$ the virtual dry static energy and $s_{\mathrm{vp}}(\lambda)$ its in-plume value. The closure relies on the fact that the time derivative of $A$ can be decomposed as, ${ }^{32}$

$\frac{d A}{d t}=\left.\frac{d A}{d t}\right|_{\mathrm{LS}}+\left.\frac{d A}{d t}\right|_{\mathrm{C}} \equiv \dot{A}_{\mathrm{LS}}+\dot{A}_{\mathrm{C}}$

where the subscripts LS and C refer to "large-scale" and "cloud" contributions, respectively.

It is worth noting that the phrase "large-scale" used by AS74 to describe the forcing of the cloud work function has been criticized (e.g. Randall et al., 1997; Mapes, 1997). Indeed, similar criticisms could be applied to the terminology of "large-scale" as used in studies of cumulus parameterization more generally. In the absence of a generally-accepted

\footnotetext{
${ }^{30} \mathrm{Eq}$. (132) of AS74

${ }^{31}$ Eq. (133) of AS74. More generally, as pointed out by Arakawa (1993), analogous closures could be based on any functional of the temperature and moisture profiles that has a threshold describing convective instability.

${ }^{32}$ Eq. (140) of AS74
}

and satisfactory alternative, however, we follow the conventional, if flawed, terminology here.

With that caveat, we wish to be very clear about the distinction between large-scale and cloud terms. In the language of normalization transformations, the distinction is entirely straightforward. $A(\lambda)$ is a normalization-invariant, and its time derivative has contributions which are normalizationinvariant (the $\dot{A}_{\mathrm{LS}}$ part) and which are globally-rescaled (the $\dot{A}_{\mathrm{C}}$ part). Thus, timescales $\tau_{\mathrm{LS}}$ and $\tau_{\mathrm{adj}}$ are appropriate for $\dot{A}_{\mathrm{LS}}$ and $\dot{A}_{\mathrm{C}}$, respectively. The physical constraint imposed is the separation of those timescales, $\tau_{\mathrm{LS}} \gg \tau_{\mathrm{adj}}$, which defines the AS74 quasi-equilibrium closure, $d A / d t \approx 0$. The closure transformation $\mathcal{T}(\lambda)$ can be constructed ${ }^{33}$ by applying this constraint to Eq. (49).

\subsection{Equivalent AS74 closure for a bulk system?}

We now consider whether an equivalent AS74 closure can be developed for the corresponding bulk model. Summing over all plumes (or equivalently, integrating over all $\lambda$ ), the kinetic energy equation (Eq. 47) becomes

$$
\frac{\partial K}{\partial t}=A_{B} M\left(z_{\text {base }}\right)-\text { DIS }
$$

where

$K=\int \mathcal{K} d \lambda ;$ DIS $=\int \mathcal{D} d \lambda$

$$
\begin{aligned}
& A_{B} \equiv \frac{\int \mathcal{M}\left(z_{\text {base }}, \lambda\right) A(\lambda) d \lambda}{M\left(z_{\text {base }}\right)} \\
& =\int_{z_{\text {base }}}^{z_{\text {TOP }}} \frac{g}{c_{p} \bar{T}} \frac{M}{M\left(z_{\text {base }}\right)}\left(s_{v B}-\overline{s_{v}}\right) d z
\end{aligned}
$$

We have introduced $z_{\mathrm{TOP}}=z_{D}(0)$ to denote the highest detrainment layer (i.e., that for a non-entraining plume of $\lambda=0$ ), and have made use of the understanding that there are no contributions to $M(z)$ from plumes characterised by a $\lambda$ such that $z>z_{D}(\lambda)$.

The bulk-cloud work function $A_{B}$, itself a global invariant, has a time derivative that cannot be decomposed into normalization-invariant and globally-rescaled parts. For example, one contribution to the time derivative is

$$
\begin{aligned}
& \frac{d A_{B}}{d t}=\int \frac{\mathcal{M}\left(z_{\text {base }}, \lambda\right)}{M\left(z_{\text {base }}\right)} \frac{d A(\lambda)}{d t} d \lambda+\cdots \\
& =\frac{\int \mathcal{M}\left(z_{\text {base }}, \lambda\right)\left(\dot{A}_{\mathrm{LS}}(\lambda)+\dot{A}_{\mathrm{C}}(\lambda)\right) d \lambda}{M\left(z_{\text {base }}\right)}+\cdots
\end{aligned}
$$

The globally-rescaled variable $\dot{A}_{\mathrm{C}}$ produces a globallyrescaled contribution to $d A_{B} / d t$, associated with timescale

\footnotetext{
${ }^{33}$ In fact, although the constraint can usually be satisfied, it is not always capable of acting as a generator, as discussed by Lord and Arakawa (1982), Lord et al. (1982).
} 
$\tau_{\text {adj }}$. The normalization-invariant variable $\dot{A}_{\mathrm{LS}}(\lambda)$, however, does not produce normalization-invariant contributions to $d A_{B} / d t$. For example, $\dot{A}_{\mathrm{LS}}(\lambda)$ includes a term proportional to changes in mixed-layer moist static-energy $h_{\mathrm{M}}$, and this leads to contributions to $d A_{B} / d t$ that include

$\frac{d A_{B}}{d t}=\frac{g}{c_{p} M\left(z_{\text {base }}\right)} \frac{\partial h_{\mathrm{M}}}{\partial t} \int d \lambda \mathcal{M}\left(z_{\text {base }}, \lambda\right)$

$\int_{z_{\mathfrak{c}}(\lambda)}^{z_{D}(\lambda)} d z^{\prime} \frac{1}{\bar{T}\left(z^{\prime}\right)}\left(\frac{1+\gamma\left(z^{\prime}\right) \epsilon\left(z^{\prime}\right) \delta}{1+\gamma\left(z^{\prime}\right)}\right)+\cdots$

Although the integral over $z^{\prime}$ has an integrand that is normalization invariant, its limits are functions of $\lambda$. Thus, the contribution is globally-invariant, and cannot be evaluated without knowledge of the full plume spectrum.

The time-derivative of $A_{B}$ can in fact be decomposed into globally-invariant and globally-rescaled parts, such that its stationarity could be used to close a bulk parameterization given a constraint that $\tau_{\text {spec }} \gg \tau_{\text {adj }}$. Such a closure would satisfy condition (1) from Sect. 6. It is unclear, however, whether $A_{B}$ can be considered to be slowly-varying in this sense. Certainly, the imposed AS74 physical constraint of $d A(\lambda) / d t \approx 0$ is no guarantee that $d A_{B} / d t \approx 0$, and so stationarity of $A_{B}$ does not satisfy condition (2) for a valid equivalent closure of a bulk parameterization.

\subsection{CAPE closure of AS74 system?}

We have shown that the bulk cloud work function may not be used to close a bulk parameterization in a manner equivalent to the AS74 quasi-equilibrium closure of a spectral parameterization. However, there may be multiple ways in which a generator to close a spectral parameterization can be reduced to a generator to close a bulk parameterization. Let us consider the undilute CAPE, or in other words, the cloud work function for a non-entraining plume,

$\mathrm{CAPE}=A(0)=\int_{z_{\text {base }}}^{z_{\mathrm{TOP}}^{\mathrm{TOP}}} \frac{g}{c_{p} \bar{T}}\left(s_{\mathrm{vp}}(0)-\overline{s_{v}}\right) d z$

The kinetic energy of non-entraining plumes is described by Eq. (47) and the decomposition of $d A(0) / d t$ from Eq. (49) applies. Clearly then a CAPE closure using $d A(0) / d t \approx 0$ is physically based upon $\tau_{\mathrm{LS}} \gg \tau_{\text {adj }}$ and so would satisfy condition (2) for equivalent closure of a bulk parameterization. However, we need to consider also condition (1): whether CAPE closure can act as a generator for a global transformation to allow determination of $M\left(z_{\text {base }}\right)$.

We examine first $\dot{A}_{\mathrm{LS}}(0)$, the normalization-invariant part of $d A(0) / d t$. One of the contributions to this is analogous to the term shown explicitly in Eq. (54) and is specifically

$$
\dot{A}_{\mathrm{LS}}(0)=\frac{g}{c_{p}} \frac{\partial h_{\mathrm{M}}}{\partial t} \int_{z_{\mathrm{LCL}}}^{z_{\mathrm{TOP}}} d z^{\prime} \frac{1}{\bar{T}\left(z^{\prime}\right)}\left(\frac{1+\gamma\left(z^{\prime}\right) \epsilon\left(z^{\prime}\right) \delta}{1+\gamma\left(z^{\prime}\right)}\right)+\cdots
$$

The explicit form of terms in $d A(0) / d t$ would not normally be used in a parameterization. However, in order for a CAPEbased closure to satisfy condition (1), then it must be possible in principle to evaluate all such terms directly using a bulk model. Examination of all such terms (not shown) reveals that this is indeed the case for $\dot{A}_{\mathrm{LS}}(0)$, provided that $z_{\mathrm{LCL}}$ is known by the bulk model. This is required to evaluate the integral in Eq. (56) for instance. In Appendix A we demonstrate that under normal conditions $z_{\mathrm{c}} \geq z_{\mathrm{LCL}}$, and so $z_{\mathrm{LCL}}$ is simply the lowest height for which $l_{B} \neq 0$. This inequality is important and explains why it is necessary to use CAPE: it would not be valid according to condition (1) to try to close a bulk parameterization using the cloud work function for any non-zero value of $\lambda$.

Consider now $\dot{A}_{\mathrm{C}}(0)$, the globally-rescaled part of $d A(0) / d t$. This can be categorized into mixed-layer terms, vertical mass-flux terms and detrainment terms. ${ }^{34}$ The mixed-layer terms can be evaluated from the environmental sounding and the total updraft-base mass flux $M\left(z_{\text {base }}\right)$, while the vertical mass-flux terms require knowledge of the full function $M(z)$. The detrainment terms include the following contribution

$$
\dot{A}_{\mathrm{C}}(0)=\frac{g L}{c_{p}} \int_{z_{\mathrm{LCL}}}^{z_{\mathrm{TOP}}} d z \frac{1}{\rho \bar{T}} D(z)[1-(1+\delta) \epsilon] \widehat{l}+\cdots
$$

This requires the detrainment profile $D(z)$ and the quantity $\widehat{l}$. The latter is problematic for a bulk parameterization, because it should properly be computed by integrating the budget equations for a single plume (Sect. 3.1). Thus, the stationarity of CAPE does not satisfy condition (1) for a valid equivalent closure of a bulk parameterization. ${ }^{35}$ The problem can be avoided by invoking again the ansatz of Eq. (20) that was introduced in Sect. 3.2 in order to formulate detrainment in the bulk-plume budget equations. We have shown then that the ansatz is required not only to compute the vertical profile of the bulk plume but that it is also necessary to permit CAPE closure ${ }^{36}$ of a bulk parameterization. The practical impact of the ansatz on closure calculations is difficult to discern: certainly this author is unaware of any attempt in the literature to assess the impact.

\footnotetext{
${ }^{34}$ See Eqs. (141), (144) and (B35) of AS74.

${ }^{35}$ In a re-derivation of the AS74 model by the present author, some additional terms in $\dot{A}_{\mathrm{C}}(0)$ were obtained that do not appear in AS74. These are proportional to the microphysical quantity $d(z, \lambda)$ defined by Eq. (B20) of AS74, and one such term also involves $\widehat{l}$. However, none of these terms affect any of the arguments presented on the formal validity of CAPE closure.

${ }^{36}$ Instead of using a cloud work function, some recent authors (e.g Kain et al., 2003; Kain, 2004; Zhang, 2009) have investigated the use of dilute CAPE for the closure of bulk parameterization. Dilute CAPE differs from CAPE by substituting $s_{\mathrm{VB}}$ for $s_{\mathrm{vp}}(0)$ in Eq. 55, or equivalently, from $A_{B}$ by omitting the factor $\mathcal{M} / M\left(z_{\text {base }}\right)$ from the integrand in Eq. 53. The use of a dilute CAPE does not alter the main argument presented in this section.
} 


\subsection{Other closures}

We have considered in some detail the AS74 quasiequilibrium closure for spectral models of entraining plumes. However, many other closures have been proposed for convective parameterizations, at least in part because any given closure may appear more or less plausible over different locations and with different grid-box sizes in the parent model (e.g. Grell et al., 1991; Grell, 1993). It would neither be practical nor instructive to consider every one, but some remarks on how other types of closure might apply to bulk and spectral parameterizations would seem to be in order.

Various authors have suggested various classifications of closure assumptions, but Grell et al.'s (1991, p. 6) is the most appropriate for our present purposes. ${ }^{37}$ Closures seek to relate the overall convective transport to: (i) a measure of largescale instability, by imposing an adjustment of that measure; (ii) a measure of large-scale advection, typically horizontal mass or moisture convergence; or, (iii) a measure of the rate of environmental destabilization. The AS74 closure is of class (iii), constraining the generation of a verticallyintegrated instability measure. Other closures with a similar basis (e.g. Moorthi and Suarez, 1992; Pan and Randall, 1998; Byun and Hong, 2007) will also have formal difficulties if applied to a bulk parameterization. The key point of difficulty for bulk models is the detrainment of condensate, and this will enter into considerations of the rate of change of environmental instability if a vertically-integrated measure encompasses the detrainment layer of any plume within the ensemble.

Closures in class (i) are popular particularly in mesoscale models and for mid-latitude applications (e.g. Frank, 1983). Typically, such a closure aims to remove CAPE, sometimes instantaneously upon convective triggering but more commonly within some "closure timescale", which is just the $\tau_{\text {adj }}$ of Sect. 5 (e.g. Fritsch and Chappell, 1980; Emanuel, 1993; Zhang and McFarlane, 1995; Gregory, 1997; Willett and Milton, 2006; Bechtold et al., 2001; Kain, 2004). This method is inspired by observations in which the triggering of a convective episode does indeed consume preexisting instability (e.g. Fritsch et al., 1976; Song and Frank, 1983). The removal is described by Eq. (49) for $\lambda=0$, and therefore the issue raised in Sect. 6.3 also applies to such closures. For a bulk parameterization, the removal of CAPE is not a well-defined closure unless one invokes the YEC73 ansatz.

Conceptually, the closures in class (ii) (e.g. Kuo, 1974; Tiedtke, 1989; Frank and Cohen, 1987; Brown, 1979) use empirical relationships between $M\left(z_{\text {base }}\right)$ and various measures of large-scale advection. Thus, they generate global

\footnotetext{
${ }^{37}$ It is not always entirely clear that a particular closure belongs uniquely to a particular class. For example, McBride (1981) showed that the AS74 closure is actually strongly dependent on horizontal mass convergence, and its vertical distribution. See also Arakawa (2004). Nonetheless, the classification is adequate for our discussion.
}

transformations that can in principle be applied freely to bulk and spectral parameterizations alike. Not seeking to revisit such debates here, we simply note that closures from this class have become markedly less popular over recent years, not least as a result of attacks on their conceptual basis from Emanuel (1994); Raymond and Emanuel (1993); Arakawa (2004) and others.

Our discussion has focussed on the formal validity (or otherwise) of the generators of global transformations for bulk parameterizations. It is important, however, that the reader should not be left with an impression that closure of a spectral parameterization is a simple matter. A physical constraint that can act as a global transformation generator is sufficient to close a bulk parameterization, but would provide none of the necessary information to a spectral parameterization about the spectral distribution of mass flux. Some spectral parameterizations apply constraints to generate explicitly a suitable normalization transformation (Arakawa and Schubert, 1974; Nober and Graf, 2005), while others combine instead a global transformation with some additional constraints to set the spectral distribution, whether by appeal to observations (e.g. Donner, 1993), or theory (e.g. Plant and Craig, 2008), or even "mainly for the sake of simplicity" (e.g. Zhang and McFarlane, 1995, p. 412). Regardless of the approach taken, setting the spectral distribution is not trivial.

\section{Conclusions}

Key aspects of climate models, for example the moisture structure in the tropics (e.g. Gregory, 1997), are highly sensitive to the formulation of entrainment in the convection parameterization (e.g. Knight et al., 2007). In a spectral model of plumes simple treatments are generally used for the entrainment into a single plume, but these become translated into overall $E(z)$ and $D(z)$ that are complicated functions of the environment. Such functions would be difficult to specify directly, and AS74 claim in effect that this makes a spectral formulation the natural choice. In a bulk model, $E(z)$ and $D(z)$ are chosen by the modeller, ${ }^{38}$ often with some switching of the functional forms between "types" (e.g. Gregory, 1997) according to the large-scale regime. Thus, a bulk parameterization offers the modeller more direct control over its behaviour. Whether this is considered to be a good or a bad point is to some extent an issue of the modelling philosophy.

\footnotetext{
${ }^{38}$ Relatively sophisticated treatments of entrainment and detrainment have been used in some bulk parameterizations (e.g. Kain and Fritsch, 1990; Emanuel, 1993), based on the "buoyancy-sorting" concept of Raymond and Blyth (1986). Such treatments do not regard the bulk plume as homogeneous but rather as being composed of air parcels that have undergone different degrees of mixing with the environment. Conceptually then, they could be viewed as attempts to use a spectral mode of thinking in order to construct complicated $E(z)$ and $D(z)$ on-the-fly.
} 
The differences between bulk and spectral parameterizations are perhaps most often thought about in terms of the specification of entrainment and detrainment, but there are also differences in the underlying theoretical structure. The theoretical differences have been the subject of this article. Budget equations for individual and for bulk plumes can be cast into very similar forms (Sect. 4) provided that an ansatz is made for the detrainment of condensate from the bulk plume. The ansatz is that $l_{D i}=l_{B}$ (Eq. 20) and is the price paid for the simplification to a single bulk plume. Moreover, similarity between the equation sets requires a very simple representation of the microphysics. The use of more complicated microphysics in bulk convective parameterizations lacks a sound theoretical basis.

While Yanai et al. (1973) are clear about the arbitrary, but convenient, nature of their ansatz, that is not always the case in later works. For example, one issue for convective parameterization is the coupling to stratiform cloud. Motivated by considerations of mesoscale organization, some authors (e.g. Frank and Cohen, 1987; Kain, 2004; Kreitzberg and Perkey, 1976) have taken a so-called "hybrid approach" (Molinari and Dudek, 1992), in which (a fraction of) the detrained condensate is acted upon by the parent model's large-scale cloud equations, allowing it to act as a source term for prognostic respresentations of stratiform cloud (e.g. Fowler et al., 1996; Tiedtke, 1993). Such treatments can have significant effects: for example, on cirrus and on the hydrological cycle in the tropics (e.g. Tiedtke, 1993; Liu et al., 2001). In the opinion of this author, however, much of the relevant literature does not seem to appreciate fully, or sometimes even to recognize, Yanai et al.'s (1973) ansatz: while the detrained condensate is predicted by a spectral parameterization, the values obtained from a bulk parameterization are systematic overestimates that by construction are not intended to be reliable.

Another consequence of Yanai et al.'s (1973) ansatz is that virtual temperature effects must be approximated or even ignored in determining the bulk plume top (Sect. 3). Moreover, there is not necessarily an equivalence between closure constraints applied to spectral and bulk parameterizations.

Closures based on CAPE, or a cloud-work function, assume a timescale separation between the slow mechanisms of atmospheric destablization and the relatively fast mechanisms of the convective response. The definition and interpretation of the slow and fast timescales has been much debated. In Sect. 5 we introduced a normalization transformation, and argued that the behaviour of a variable under such a transformation is sufficient to associate that variable with a well-defined timescale. We were then able to show that the quasi-equilibrium closure for spectral parameterizations introduced by Arakawa and Schubert (1974) does not correspond in any straightforward way to a suitable closure constraint for bulk parameterizations. The natural analogue to the Arakawa and Schubert (1974) closure is the stationarity of the bulk cloud work function, but the evolution of this variable is governed by timescales $\tau_{\text {spec }}$ and $\tau_{\text {adj }}$ (Sect. 6.2), rather than the timescales $\tau_{\mathrm{LS}}$ and $\tau_{\text {adj }}$ governing the evolution of the cloud work function $A(\lambda)$ (Sect. 6.1). Thus, the stationarity of $A_{B}$ and $A(\lambda)$ would encapsulate distinct physical constraints. $\tau_{\text {spec }}$ and $\tau_{\text {LS }}$ do not seem to have been clearly distinguished before now, let alone studied in any systematic way.

This timescale issue can be avoided if one closes a bulk parameterization using either the removal of CAPE by the plume ensemble or a quasi-equilibrium constraint of $d \mathrm{CAPE} / d t \approx 0$. It should be noted, however, that a computation of $d \mathrm{CAPE} / d t$ involves the detraining condensate from each plume, and so cannot be performed by a bulk model, unless the Yanai et al. (1973) ansatz is used. Thus, the ansatz is a necessary ingredient in such a closure (Sect. 6.3). Whether this has a practical impact on the closure of bulk parameterizations has not been examined in the literature.

In comparing a "full" and a "simplified" physical model, there is always a danger of confusing complexity with sophistication. Most convective parameterizations in use today are of the bulk form, and this is undoubtedly a convenient simplification that should not be discarded lightly. It is obtained by invoking Yanai et al.'s (1973) ansatz and has implications for: the microphysics of convective and associated layer cloud; the calculation of cloud top; and, the validity of closure methods for bulk parameterization. Some of those implications were previously known, but perhaps obscure, whereas others have been raised here. The extent to which such theoretical issues with the structure of bulk parameterizations may affect their actual performance in practice is not well studied, but systematic investigations are required if modellers are to make well-informed judgements about the continued use of bulk parameterizations. The question to be continually asked is not so much is a bulk or a spectral method to be preferred? but rather is the bulk framework conceptually "good enough" for our present and future purposes?.

\section{Appendix A}

\section{Cloud base level}

The purpose of this Appendix is to demonstate that under normal atmospheric conditions then $z_{\mathrm{c}} \geq z_{\mathrm{LCL}}$, as stated in Sect. 6.3: i.e., that cloud base for an entraining plume lies above that for a non-entraining plume.

Cloud base is defined in AS74 to be the lowest height at which Eq. (8) is satisfied, describing the saturation of inplume air. It is convenient to restate that equation here,

$s_{\mathrm{p}}-\bar{s} \approx \frac{1}{1+\gamma}\left(h_{\mathrm{p}}-\bar{h}^{*}\right)$ for $z \geq z_{\mathrm{c}}(\lambda)$

in which $s_{\mathrm{p}}$ and $h_{\mathrm{p}}$ are obtained by integrating the plume budget equations upwards from the updraft base with ini- 
tial conditions ${ }^{39}$ taken from the mixed-layer properties, $s_{\mathrm{p}}\left(z_{\text {base }}\right)=s_{\mathrm{M}}$ and $h_{\mathrm{p}}\left(z_{\text {base }}\right)=h_{\mathrm{M}}$. For a non-entraining plume $s_{\mathrm{p}}$ and $h_{\mathrm{p}}$ retain their initial values and so $z_{\mathrm{LCL}}$ is defined by the lowest height which satisfies

$s_{\mathrm{M}}-\bar{s} \approx \frac{1}{1+\gamma}\left(h_{\mathrm{M}}-\bar{h}^{*}\right)$ for $z \geq z_{\mathrm{LCL}}$

Now, given two equations $g_{1}\left(z_{1}\right)=0$ and $g_{2}\left(z_{2}\right)=0$ then if $g_{2} \approx g_{1}$, a simple Taylor series expansion of $g_{2}$ about $z_{1}$ yields

$z_{1}-z_{2}=\frac{g_{2}\left(z_{1}\right)-g_{1}\left(z_{1}\right)}{g_{2}^{\prime}\left(z_{1}\right)}$

the dash here denoting a vertical derivative. Applying this to the above equations defining cloud base, we have

$z_{\mathrm{c}}-z_{\mathrm{LCL}}=\left.\frac{\gamma\left(s_{\mathrm{p}}-s_{\mathrm{M}}\right)-L\left(q_{\mathrm{p}}-q_{\mathrm{M}}\right)}{g \gamma+\bar{s} \gamma^{\prime}}\right|_{z_{\mathrm{c}}}$

where we have used the definitions of $\gamma$ and $\bar{s}$ to simplify the denominator. For water vapour both $\gamma$ and $\gamma^{\prime}$ are positive, and so the denominator must be positive. Thus, if the numerator is also positive, then $z_{\mathrm{c}} \geq z_{\mathrm{LCL}}$ as required.

Consider the two bracketed terms in the numerator. Assuming that $\bar{s}$ increases monotonically with height between $z_{\text {base }}$ and $z_{\mathrm{c}}$ (i.e., that the lapse rate is no stronger than dry adiabatic), then the entrainment process must produce values of $s_{\mathrm{p}}$ that are larger than $s_{\mathrm{M}}$. Similarly a monotonic decrease of $q$ within the environment must produce $q_{\mathrm{p}}\left(z_{\mathrm{c}}\right)<q_{\mathrm{M}}$. Under normal atmospheric conditions therefore, the numerator is indeed positive.

\section{Appendix B}

\section{Nomenclature: Symbols not explicitly listed below have their standard meteorological meanings.}

\begin{tabular}{ll}
\hline$\chi_{\mathrm{vc}}$ & subscript denoting a virtual contribution \\
$\chi_{B}$ & subscript denoting a bulk value \\
$\chi_{i}$ & subscript denoting a specific plume \\
$\chi_{\mathrm{C}}$ & subscript denoting a cloud term \\
$\chi_{\mathrm{LS}}$ & subscript denoting a large-scale term \\
$\chi_{\mathrm{M}}$ & subscript denoting a mixed-layer value \\
$\chi_{\mathrm{p}}$ & subscript denoting an in-plume value \\
$\chi_{D i}$ & subscript denoting value on detrainment from \\
& plume $i$ \\
$\chi^{\prime}$ & prime denoting deviation from horizontal mean \\
$\chi^{*}$ & superscript denoting saturated value \\
$\chi^{d}$ & superscript denoting a quantity to be evaluated \\
& in the detraining layers of contributing plumes \\
& only \\
\hline
\end{tabular}

\footnotetext{
${ }^{39}$ Eqs. (129) and (131) of AS74
}

\begin{tabular}{|c|c|}
\hline$\chi^{e}$ & $\begin{array}{l}\text { superscript denoting a quantity to be evaluated } \\
\text { in the entraining layers of contributing plumes } \\
\text { only }\end{array}$ \\
\hline$\dot{\chi}$ & time derivative of $\chi$ \\
\hline $\bar{\chi}$ & horizontally-averaged value of $\chi$ \\
\hline$\widehat{\chi}$ & value at the detrainment level \\
\hline$\tilde{\chi}$ & environmental value of $\chi$ \\
\hline$\delta$ & $\begin{array}{l}\text { thermodynamic parameter: one less than the ra- } \\
\text { tio of gas constants of water vapour to dry air }\end{array}$ \\
\hline$\epsilon$ & thermodynamic parameter defined by Eq. (13) \\
\hline$\gamma$ & thermodynamic parameter defined by Eq. (10) \\
\hline$\lambda$ & parameter defining an entraining plume type \\
\hline$\sigma$ & fractional area covered by plume \\
\hline$\tau_{\lambda}$ & timescale for plume type $\lambda$ \\
\hline$\tau_{\mathrm{adj}}$ & $\begin{array}{l}\text { adjustment timescale for overall amplitude of } \\
\text { convective activity }\end{array}$ \\
\hline$\tau_{\mathrm{LS}}$ & large-scale timescale \\
\hline$\tau_{\text {spec }}$ & $\begin{array}{l}\text { timescale for changes to spectral distribution } \\
\text { under a fixed large-scale condition }\end{array}$ \\
\hline $\mathcal{D}$ & rate of kinetic energy dissipation \\
\hline $\mathcal{K}$ & kinetic energy \\
\hline $\mathcal{T}$ & normalization transformation \\
\hline$A$ & cloud work function \\
\hline$c$ & rate of condensation \\
\hline$C_{0}$ & $\begin{array}{l}\text { constant defining the autoconversion rate in the } \\
\text { system of AS74, via Eq. (44) }\end{array}$ \\
\hline$D$ & detrainment rate \\
\hline$E$ & entrainment rate \\
\hline$e$ & evaporation rate \\
\hline$h$ & moist static energy \\
\hline$K$ & total kinetic energy of plume ensemble \\
\hline$k$ & $\begin{array}{l}\text { empirical function defining the autoconversion } \\
\text { rate in the system of YEC73, via Eq. (34) }\end{array}$ \\
\hline$l$ & plume liquid water \\
\hline$M$ & convective mass flux \\
\hline$Q_{\mathrm{R}}$ & radiative heating rate \\
\hline$R$ & conversion rate \\
\hline$s$ & dry static energy \\
\hline$s_{v}$ & virtual dry static energy \\
\hline$s_{\mathrm{vp}}$ & in-plume value of virtual dry static energy \\
\hline$z_{\text {base }}$ & base level for entraining plumes \\
\hline$z_{\mathrm{LCL}}$ & lifting condensation level \\
\hline$z_{\mathrm{TOP}}$ & $\begin{array}{l}\text { highest detrainment level from the plume en- } \\
\text { semble }\end{array}$ \\
\hline$z_{D}$ & detrainment level \\
\hline$z_{\mathrm{c}}$ & cloud base level \\
\hline DIS & total dissipation rate of plume ensemble \\
\hline
\end{tabular}

Acknowledgements. A conversation with P. Siebesma and J.-I. Yano at the Workshop on Concepts for Convective Parameterizations in Large-Scale Models first inspired me to delve into these issues.

Edited by: J. Quaas 


\section{References}

Arakawa, A.: Closure assumptions in the cumulus parameterization problem, in: The Representation of Cumulus Convection in $\mathrm{Nu}-$ merical Models, edited by: Emanuel, K. A. and Raymond, D. J., of Meteorological Monographs, chap. 1, Am. Meteorol. Soc., 24, 1-16, 1993.

Arakawa, A.: The cumulus parameterization problem: past, present and future, J. Climate, 17, 2493-2525, 2004.

Arakawa, A. and Schubert, W. H.: Interaction of a cumulus cloud ensemble with the large-scale environment, Part I, J. Atmos. Sci., 31, 674-701, 1974.

Bechtold, P., Bazile, E., Guichard, F., and Richard, E.: A mass-flux convection scheme for regional and global models, Q. J. Roy. Meteor. Soc., 127, 869-886, 2001.

Betts, A. K. and Miller, M. J.: A new convective adjustment scheme, Part II: single column tests using GATE wave, BOMEX, ATEX and arctic air-mass data sets, Q. J. Roy. Meteor. Soc., 112, 793-809, 1986.

Bougeault, P.: A simple parameterization of the large-scale effects of cumulus convection, Mon. Weather Rev., 113, 2108-2121, 1985.

Brown, J. M.: Mesoscale unsaturated downdrafts driven by rainfall evaporation: a numerical study, J. Atmos. Sci., 36, 313-338, 1979.

Byun, Y.-H. and Hong, S.-Y.: Improvements in the subgrid-scale representation of moist convection in a cumulus parameterization scheme: the single-column test and its impact on seasonal prediction, Mon. Weather Rev., 135, 2135-2154, 2007.

Cho, H.-R.: Contributions of cumulus cloud life-cycle effects to the large-scale heat and moisture budget equations, J. Atmos. Sci., 34, 87-97, 1977.

Cohen, B. G. and Craig, G. C.: The response time of a convective cloud ensemble to a change in forcing, Q. J. Roy. Meteor. Soc., 130, 933-944, 2004.

Donner, L. J.: A cumulus parameterization including mass fluxes, vertical momentum dynamics, and mesoscale effects, J. Atmos. Sci., 50, 889-906, 1993.

Emanuel, K. A.: A cumulus representation based on the episodic mixing model: the importance of mixing and microphysics in predicting humidity, in: The Representation of Cumulus Convection in Numerical Models, edited by: Emanuel, K. A. and Raymond, D. J. of Meteorological Monographs, Am. Meteorol. Soc., 19(24), 185-192, 1993.

Emanuel, K. A.: Atmospheric Convection, Oxford University Press, 1994.

Esbensen, S.: Bulk thermodynamic effects and properties of small tropical cumuli, J. Atmos. Sci., 35, 826-837, 1978.

Fowler, L. D., Randall, D. A., and Rutledge, S. A.: Liquid and ice cloud microphysics in the CSU general circulation model, Part 1: model description and simulated microphysical processes, J. Climate, 9, 489-529, 1996.

Frank, W. M.: The cumulus parameterization problem, Mon. Weather Rev., 111, 1859-1871, 1983.

Frank, W. M. and Cohen, C.: Simulation of tropical convective systems, Part I: a cumulus parameterization, J. Atmos. Sci., 44, 3787-3799, 1987.

Fritsch, J. M. and Chappell, C. F.: Numerical prediction of convectively driven mesoscale pressure systems, Part I: convective parameterization, J. Atmos. Sci., 37, 1722-1733, 1980.
Fritsch, J. M. and Kain, J. S.: Convective parameterization for mesoscale models: the Fritsch-Chappell scheme, in: The Representation of Cumulus Convection in Numerical Models, edited by: Emanuel, K. A. and Raymond, D. J. of Meteorological Monographs, Am. Meteorol. Soc., 24(15), 159-164, 1993.

Fritsch, J. M., Chappell, C. F., and Hoxit, L. R.: The use of largescale budgets for convective parameterization, Mon. Weather Rev., 104, 1408-1418, 1976.

Garner, S. T., Frierson, D. M. W., Held, I. M., Pauluius, O., and Vallis, G. K.: Resolving convection in a global hypohydrostatic model, J. Atmos. Sci., 64, 2061-2075, 2007.

Gerard, L. and Geleyn, J.-F.: Evolution of a subgrid deep convection parameterization in a limited-area model with increasing resolution, Q. J. Roy. Meteorol. Soc., 131, 2293-2312, 2005.

Gregory, D.: The mass flux approach to the parameterization of deep convection, in: The Physics and Parameterization of Moist Atmospheric Convection, edited by: Smith, R. K., Kluwer Academic Publishers, 297-319, 1997.

Gregory, D. and Rowntree, P. R.: A mass flux convection scheme with representation of cloud ensemble characteristics and stability-dependent closure, Mon. Weather Rev., 118, 14831506, 1990.

Grell, G. A.: Prognostic evaluation of assumptions used by cumulus parameterizations, Mon. Weather Rev., 121, 764-787, 1993.

Grell, G. A., Kuo, Y.-H., and Pasch, R. J.: Semiprognostic tests of cumulus parameterization schemes in the middle latitudes, Mon. Weather Rev., 119, 5-31, 1991.

Hack, J. J., Schubert, W. H., and Dias, P. L. S.: A spectral cumulus parameterization for use in numerical models of the tropical atmosphere, Mon. Weather Rev., 112, 704-716, 1984.

Johnson, R. H.: The role of convective-scale precipitation downdrafts in cumulus and synoptic-scale interactions, J. Atmos. Sci., 33, 1890-1910, 1976.

Johnson, R. H.: The effects of cloud detrainment on the diagnosed properties of cumulus populations, J. Atmos. Sci., 34, 359-366, 1977.

Johnson, R. H. and Young, G. S.: Heat and moisture budgets of tropical mesoscale anvil clouds, J. Atmos. Sci., 40, 2138-2147, 1983.

Jordan, C. L.: Mean soundings for the West Indies area, J. Meteorol., 15, 91-97, 1958.

Kain, J. S.: The Kain-Fritsch convective parameterization: an update, J. Appl. Meteorol., 43, 170-181, 2004.

Kain, J. S. and Fritsch, J. M.: A one-dimensional entraining/detraining plume model and its application in convective parameterization, J. Atmos. Sci., 47, 2784-2802, 1990.

Kain, J. S., Fritsch, J. M., and Weiss, S. J.: Parameterized updraft mass flux as a predictor of convective intensity, Weather Forecast., 18, 106-116, 2003.

Khairoutdinov, M., Randall, D., and DeMott, C.: Simulations of the atmospheric general circulation using a cloud-resolving model as a superparameterization of physical processes, J. Atmos. Sci., 62, 2136-2154, 2005.

Knight, C. G., Knight, S. H. E., Massey, N., Aina, T., Christensen, C., Frame, D. J., Kettleborough, J. A., Martin, A., Pascoe, S., Sanderson, B., Stainforth, D. A., and Allen, M. R.: Association of parameter, software, and hardware variation with large-scale behavior across 57000 climate models, P. Natl. Acad. Sci., 104, 12259-12264, 2007. 
Kreitzberg, C. W. and Perkey, D. J.: Release of potential instability: Part I. A sequential plume model within a hydrostatic primitive equation model, J. Atmos. Sci., 33, 456-475, 1976.

Kuang, Z. and Bretherton, C. S.: A mass-flux scheme view of a high-resolution simulation of a transition from shallow to deep convection, J. Atmos. Sci., 63, 1895-1909, 2006.

Kuo, H.-L.: Further studies of the parameterization of the influence of cumulus convection on large-scale flow, J. Atmos. Sci., 31, 1232-1240, 1974.

Lawrence, M. G. and Rasch, P. J.: Tracer transport in deep convective updrafts: plume ensemble versus bulk formulations, J. Atmos. Sci., 62, 2880-2894, 2005.

Lin, C.: Some bulk properties of cumulus ensembles simulated by a cloud-resolving model, Part II: entrainment profiles, J. Atmos. Sci., 56, 3736-3748, 1999.

Lin, C. and Arakawa, A.: The macroscopic entrainment processes of simulated cumulus ensemble, Part II: testing the entrainingplume model, J. Atmos. Sci., 54, 1044-1053, 1997.

Liu, C., Moncrieff, M. W., and Grabowski, W. W.: Explicit and parameterized realizations of convective cloud systems in TOGA COARE, Mon. Weather Rev., 121, 1689-1703, 2001.

Lord, S. J. and Arakawa, A.: Interaction of a cumulus cloud ensemble with the large-scale environment, Part III: semi-prognostic tests of the Arakawa-Schubert cumulus parameterization, J. Atmos. Sci., 39, 88-103, 1982.

Lord, S. J., Chao, W. C., and Arakawa, A.: Interaction of a cumulus cloud ensemble with the large-scale environment, Part IV: the discrete model, J. Atmos. Sci., 39, 104-113, 1982.

Mapes, B. E.: Equilibrium vs. activation control of large-scale variations of tropical deep convection, in: The Physics and Parameterization of Moist Atmospheric Convection, edited by: Smith, R. K., Kluwer Academic Publishers, 321-358, 1997.

McBride, J. L.: An analysis of diagnostic cloud mass flux models, J. Atmos. Sci., 38, 1977-1990, 1981.

Molinari, J. and Dudek, M.: Parameterization of convective precipitation in mesoscale numerical models: a critical review, Mon. Weather Rev., 120, 326-344, 1992.

Moorthi, S. and Suarez, M. J.: Relaxed Arakawa-Schubert: a parameterization of moist convection for general circulation models, Mon. Weather Rev., 120, 978-1002, 1992.

Nitta, T.: Observational determination of cloud mass flux distributions, J. Atmos. Sci., 32, 73-91, 1975.

Nitta, T.: Response of cumulus updraft and downdraft to GATE A/B-scale motion systems, J. Atmos. Sci., 34, 1163-1186, 1977.

Nober, F. J. and Graf, H. F.: A new convective cloud field model based on principles of self-organisation, Atmos. Chem. Phys., 5, 2749-2759, 2005, http://www.atmos-chemphys.net/5/2749/2005/.

Nordeng, T. E.: Extended versions of the convective parameterization scheme at ECMWF and their impact on the mean and transient activity of the model in the tropics, Technical Memorandum 206, ECMWF, 1994.

Pan, D.-M. and Randall, D. A.: A cumulus parameterization with prognostic closure, Q. J. Roy. Meteor. Soc., 124, 949-981, 1998.

Plant, R. S. and Craig, G. C.: A shocastic parameterization for deep convection based on equilibrium statistics, J. Atmos. Sci., 65, 87-105, 2008.

Piriou, J. M., Redelsperger, J.-L., Geleyn, J.-F., Lafore, J.-P., and Guichard, F.: An approach for convective parameterization with memory: Separating microphysics and transport in grid-scale equations, J. Atmos. Sci., 64, 4127-4139, 2007.

Randall, D. A., Pan, D.-M., Ding, P., and Cripe, D. G.: QuasiEquilibrium, in: The Physics and Parameterization of Moist Atmospheric Convection, edited by: Smith, R. K., Kluwer Academic Publishers, 359-385, 1997.

Randall, D. A., Wood, R. A., Bony, S., Colman, R., Fichefet, T., Fyfe, J., Kattsov, V., Pitman, A., Shukla, J., Srinivasan, J., Stouffer, R. J., Sumi, A., and Taylor, K. E.: Climate models and their evaluation, in: Climate Change 2007: The Physical Basis, Contribution of Working Group I to the Fourth Assessment Report of the Intergovernmental Panel on Climate Change, edited by: Solomon, S., Qin, D., Manning, M., Chen, Z., Marquis, M., Averyt, K. B., Tignor, M., and Miller, H. L., Cambridge University Press, Cambridge, UK and New York, NY, USA, 2007.

Raymond, D. J. and Blyth, A. M.: A stochastic mixing model for nonprecipitating cumulus clouds, J. Atmos. Sci., 43, 2708-2718, 1986.

Raymond, D. J. and Emanuel, K. A.: The Kuo cumulus parameterization, in: The Representation of Cumulus Convection in Numerical Models, edited by: Emanuel, K. A. and Raymond, D. J. of Meteorological Monographs, Am. Meteorol. Soc., 24(10), 145-147, 1993.

Shutts, G. and Allen, T.: Sub-gridscale parametrization from the perspective of a computer games animator, Atmos. Sci. Lett., 8, 85-92, 2007.

Song, J.-L. and Frank, W. M.: Relationship between deep convection and large-scale processes during GATE, Mon. Weather Rev., 111, 2145-2160, 1983.

Straka, J. M.: Cloud and Precipitation Microphysics: Principles and Parameterizations, Cambridge University Press, 2009.

Swann, H.: Evaluation of the mass-flux approach to parametrizing deep convection, Q. J. Roy. Meteor. Soc., 127, 1239-1260, 2001.

Tiedtke, M.: A comprehensive mass flux scheme for cumulus parameterization in large-scale models, Mon. Weather Rev., 117, 1779-1800, 1989.

Tiedtke, M.: Representation of clouds in large-scale models, Mon. Weather Rev., 121, 3040-3061, 1993.

Willett, M. R. and Milton, S. F.: The tropical behaviour of the convective parameterization in aquaplanet simulations and the sensitivity to timestep, Forecasting Research Technical Report 482, Met Office, UK, 2006.

$\mathrm{Xu}, \mathrm{K} . \mathrm{M}$. and Randall, D. A.: Influence of large-scale advective colling and moistening effects on the quasi-equilibrium behavior of explicitly simulated cumulus ensembles, J. Atmos. Sci., 55, 896-909, 1998.

Yanai, M. and Johnson, R. H.: Impacts of cumulus convection on thermodynamic fields, in: The Representation of Cumulus Convection in Numerical Models, edited by: Emanuel, K. A. and Raymond, D. J. of Meteorological Monographs, Am. Meteorol. Soc., 24(4), 39-62, 1993.

Yanai, M., Esbensen, S., and Chu, J.-H.: Determination of bulk properties of tropical cloud clusters from large-scale heat and moisture budgets, J. Atmos. Sci., 30, 611-627, 1973.

Yanai, M., Chu, J.-H., Starx, T. E., and Nitta, T.: Response of deep and shallow tropical maritime cumuli to large-scale processes, J. Atmos. Sci., 33, 976-991, 1976.

Yano, J.-I., Grabowski, W. W., Roff, G. L., and Mapes, B. E.: Asymptotic approaches to convective quasi-equilibrium, Q. J. 
Roy. Meteor. Soc., 126, 1861-1887, 2000.

Yano, J.-I., Guichard, F., Lafore, J.-P., Redelsperger, J.-L., and Bechtold, P.: Estimations of mass fluxes for cumulus parameterizations from high-resolution spatial data, J. Atmos. Sci., 61, 829-842, 2004.

Zhang, G. J. and McFarlane, N. A.: Sensitivity of climate simulations to the parameterization of cumulus convection in the Canadian climate centre general circulation model, Atmos.-Ocean, 33, 407-446, 1995.
Zhang, G. J.: Effects of entrainment on convective available potential energy and closure assumptions in convection parameterization, J. Geophys. Res., 114, D07109, doi:10.1029/2008JD010976, 2009. 